\title{
Risk factors for vancomycin-resistant enterococci colonisation in critically ill patients
}

\author{
Deivid William da Fonseca Batistão ${ }^{1 /+}$, Paulo Pinto Gontijo-Filho', \\ Natália Conceiçãoº ${ }^{2}$ Adriana Gonçalves de Oliveira², Rosineide Marques Ribas ${ }^{1}$
}

'Laboratório de Microbiologia, Instituto de Ciências Biomédicas, Universidade Federal de Uberlândia, Av. Pará 1720 Bloco 4 C,

38400-902 Uberlândia, MG, Brasil '2Laboratório de Microbiologia, Universidade Federal do Triângulo Mineiro, Uberaba, MG, Brasil

Vancomycin-resistant enterococci (VRE) are important hospital pathogens and have become increasingly common in patients admitted to the intensive care unit (ICU). To determine the incidence and the risk factors associated with VRE colonisation among ICU patients, active surveillance cultures for VRE faecal carriages were carried out in patients admitted to the ICU of the University Hospital of Uberlândia, Minas Gerais, Brazil. Risk factors were assessed using a case-control study. Seventy-seven patients (23.1\%) were found to be colonised with vanC VRE and only one patient $(0.3 \%)$ was colonised with vanA VRE. Independent risk factors for VRE colonisation included nephropathy [odds ratio $(O R)=13.6, p<0.001]$, prior antibiotic use $(O R=5.5, p<0.03)$ and carbapenem use (OR $=17.3, p<0.001)$. Our results showed a higher frequency (23.1\%) of Enterococcus gallinarum and Enterococcus casseliflavus, species that are intrinsically resistant to low levels of vancomycin (vanC), without an associated infection, associated with prior antibiotic use, carbapenem use and nephropathy as comorbidity. This study is the first to demonstrate the risk factors associated with vanC VRE colonisation in ICU hospitalised patients. Although vanA and vanB enterococci are of great importance, the epidemiology of vanC VRE needs to be better understood. Even though the clinical relevance of vanC VRE is uncertain, these species are opportunistic pathogens and vanC VREcolonised patients are a potential epidemiologic reservoir of resistance genes.

Key words: risk factors - vancomycin-resistant enterococci - vanC

Vancomycin-resistant enterococci (VRE) have emerged worldwide as important nosocomial pathogens. The prevalence and incidence of VRE colonisation vary widely among hospitals and studies have suggested that such VRE rates are higher among critically ill patients, particularly those admitted to intensive care units (ICUs), limiting the therapeutic options available (Hendrix et al. 2001, Mutnick et al. 2003, Mazuski, 2008).

Glycopeptide resistance in enterococci is associated with diverse phenotypes (Werner et al. 2008) and their resistance to several antimicrobial agents, whether intrinsic (low-level resistance to penicillin, cephalosporins and aminoglycosides) or acquired (resistance to glycopeptides and high concentrations of aminoglycosides), is of great concern (Metallidis et al. 2006).

The most infectious cases are associated with Enterococcus faecalis and Enterococcus faecium; in these species, the presence of $\operatorname{van} \mathrm{A}$ and $\operatorname{van} \mathrm{B}$ genes that encode high-level vancomycin resistance is of great concern due to their spread worldwide (Mazuski 2008).

Enterococcus gallinarum and Enterococcus casseliflavus are species that are intrinsically resistant to low levels of vancomycin (vanC) and have been found to colo-

Financial support: $\mathrm{CNPq}$

+Corresponding author: deividwfb@yahoo.com.br

Received 8 April 2011

Accepted 10 October 2011 nise the human intestinal tract (Toye et al. 1997, Mondino et al. 2003, Maschieto et al. 2004). Although these species have been reported to cause infections sporadically, their clinical significance remains uncertain (Mutnick et al. 2003, de Perio et al. 2006, Neves et al. 2009).

Intestinal colonisation by VRE has been associated with a variety of risk factors and asymptomatic colonisation by VRE often precedes true infection. Although many studies on the prevalence, incidence, epidemiology and risk factors of VRE have been performed in Europe and the United States, such data for Brazil are scarce (Warren et al. 2003, Furtado et al. 2005). Moreover, despite higher rates of vanC VRE colonisation, the risk factors for colonisation by enterococci of this phenotype have not been identified (Neves et al. 2009).

The purpose of this study was to determine the incidence of VRE colonisation among patients admitted to the ICU of a tertiary teaching hospital and to identify the risk factors that predispose these hospitalised patients to VRE colonisation.

\section{PATIENTS, MATERIALS AND METHODS}

Study setting and population - The University Hospital of Uberlândia, located in the state of Minas Gerais (MG), southeastern region of Brazil, is a 503-bed tertiary teaching hospital with a 15-bed medical and surgical ICU. We performed a prospective observational study to determine the incidence of VRE colonisation among patients admitted to this ICU between April 2009-January 2010.

Rectal swabs were obtained within $48 \mathrm{~h}$ of ICU admission to detect VRE colonisation that might have occurred prior to entering the unit. Samples of patients who 
remained in the ICU for more than $48 \mathrm{~h}$ were acquired once a week until a positive VRE culture was achieved, the patient was discharged from the ICU, or the patient died. Enterococcus was considered to be ICU-acquired if it was detected from a patient who had been on the unit for more than $48 \mathrm{~h}$. Patients who had serious medical conditions that precluded the collection had left the ICU prior to collection, were non-consenting or who had nonconsenting guardians were excluded. The study protocol was approved by the hospital's ethical committee.

Isolation, identification and susceptibility testing - The samples obtained were transported to the microbiology laboratory for selective culturing of VRE. The swabs were inoculated on $\mathrm{BBL}^{\mathrm{TM}}$ Enterococcosel ${ }^{\mathrm{TM}}$ Agar plates (BD, Becton, Dickinson and Company, France), supplemented with vancomycin $(6 \mu \mathrm{g} / \mathrm{mL})$ and incubated aerobically at $35^{\circ} \mathrm{C}$ for $48 \mathrm{~h}$. All enterococci samples that were not sensitive to vancomycin were considered VRE. From each plate, some suspected enterococci colonies were initially identified by Gram staining, growth in $6.5 \% \mathrm{NaCl}$ broth and bile esculin hydrolysis. All presumed enterococci were further identified as previously described (Teixeira et al. 2007) using the following phenotypic tests: yellow-pigment production, motility, L-pirrolidonil- $\beta$-naftilamida hydrolysis, acid formation from sorbitol and arabinose and arginine hydrolysis.

Antimicrobial susceptibility testing was performed on Müeller-Hinton Agar (BD, Becton, Dickinson and Company) according to recommendations of the Clinical and Laboratory Standard Institute (CLSI 2009). The vancomycin minimal inhibitory concentration (MIC) was evaluated by the E-test method (AB Biodisk) and isolates with a MIC $\geq 32 \mu \mathrm{g} / \mathrm{mL}$ were selected for testing by polymerase chain reaction (PCR). The high level resistance to aminoglycosides was carried out by discdiffusion with gentamicin $(120 \mu \mathrm{g})$ and streptomycin $(300 \mu \mathrm{g})$ (Laborclin Ltda).

The isolates identified as E. gallinarum and E. casseliflavus with low-level resistance to vancomycin (MIC $=2-32 \mu \mathrm{g} / \mathrm{mL}$ ) were considered as the vanC phenotype.

Epidemiological investigation - A case-control study was then performed. Cases were defined as patients with VRE colonisation, but without other infections, and the controls were defined as patients with neither VRE colonisation nor infection from any microorganism. The following sociodemographic, clinical and laboratory data were evaluated: age, gender, duration of hospitalisation, prior ICU hospitalisation, underlying diseases, surgery, infections and antibiotic treatment. This information was obtained by a review of medical records and computer systems databases.

Prior antibiotic use was defined as the administration of antimicrobials in the 15 days before ICU admission. Prior ICU hospitalisation was defined as an inpatient admission to the ICU for any reason in the 12 months before the current ICU admission.

The defined daily dose (DDD) of antibiotics per 1,000 patients-day was calculated as previously described (Moreira et al. 2009).
Molecular methods - To assess the presence of the van $\mathrm{A}$ and $v a n \mathrm{~B}$ genes in enterococci strains with high MIC for vancomycin ( $\geq 32 \mu \mathrm{g} / \mathrm{mL}$ ), the PCR was performed as previously described (Woodford et al. 1993). Briefly, genomic DNA was extracted using a QIAamp DNA Mini Kit (Qiagen, Hilden, Germany). The amplification primers for vanA gene were A ATGGCAAGTCAGGTGAAGATGG and $\mathrm{A}_{2}$ TCCACCTCGCCAACAACTAACG, and for $v a n \mathrm{~B}$ gene, $\mathrm{B}_{1}$ TCTGTTTGAATTGTCTGGTAT and $\mathrm{B}_{2}$ GACCTCGTTTAGAACGATG. The cycling conditions for the PCR were as follows: 5 min at $94^{\circ} \mathrm{C}, 30$ cycles of $25 \mathrm{~s}$ at $94^{\circ} \mathrm{C}, 40 \mathrm{~s}$ at $52^{\circ} \mathrm{C}$ and $50 \mathrm{~s}$ at $72^{\circ} \mathrm{C}$ and a final step of $10 \mathrm{~min}$ at $72^{\circ} \mathrm{C}$ in a GeneAmp ${ }^{\circledR}$ PCR System 9700 (Applied Biosystems, USA). The PCR products were detected in $1.5 \%$ agarose gel by gel electrophoresis and ethidium bromide staining.

Statistical analysis - Statistical analyses were performed using GraphPad Prism v.4 (GraphPad Software, San Diego, CA) and Bioestat 5.0 (Belém, PA, Brazil). For determining the risk factors for VRE colonisation, the control and case groups were compared using the Mann-Whitney U test for continuous data and the Fisher exact test or chi-squared test for categorical data, when appropriate. We used a Pearson's correlation test to calculate the correlation between VRE colonisation per 1,000 patients-day and DDD of antibiotics per 1,000 patients-day. Multiple logistic regression was performed for the significant variables in the univariate analysis. Statistical significance was defined as $\mathrm{p} \leq 0.05$.

\section{RESULTS}

During the study period, 422 patients were admitted to the adult ICU at the University Hospital of Uberlândia. Of these patients, 328 stayed there for more than $48 \mathrm{~h}$. Eighty-nine patients were excluded for the reasons listed above. From 333 patients included in the study, $78(23.4 \%)$ were VRE-colonised (18.3/1,000 patientsday) and $35.9 \%$ (28/78) of these VRE-colonised patients had acquired the infection in the ICU. We observed that $64.1 \%(50 / 78)$ were already colonised with VRE upon ICU admission, including 46\% (23/50) who were transferred from general wards and $54 \%$ (27/50) who were from outside of the hospital.

Enterococcus spp were isolated in 90 (27\%) of the 333 patients included in this study and two of them (2.2\%) presented with more than one species of enterococci in the faecal samples, resulting in a total of 92 samples isolated. The enterococci species isolated in our study included E. casseliflavus (55.4\%), E. gallinarum (28.3\%), E. faecium (12\%) and E. faecalis (4.3\%). We isolated only one $E$. faecalis strain, which carried the vanA gene.

The distribution of antimicrobial resistance among enterococcal species is presented in Table I. The results showed that only one E. faecalis isolated was resistant to high-level vancomycin (MIC $\geq 256 \mu \mathrm{g} / \mathrm{mL}$ ). In general, the samples showed a multiresistance profile. A resistance to erythromycin was the most frequent form of antibiotic resistance (45.7\%), followed by resistance to tetracycline $(32.6 \%)$, ciprofloxacin $(17.3 \%)$ and chloramphenicol $(10.9 \%)$. Only two strains were peni- 
cillin resistant and none were ampicillin resistant. A high-level resistance to aminoglycoside was observed in $16.3 \%$ of the strains.

From 78 VRE-colonised patients, 21 of them were included in the case group, because they did not present any infection and the vanA VRE-colonised patient was excluded of the case group because acquired other infections. From 255 VRE non-colonised patients, 143 (56.1\%) had no colonisation or infection from any organism and were included in the control group. Twenty-eight VREcolonised patients were VRE-negative at admission and became colonised during their hospital stay. None of the VRE-colonised patients developed other infections during the observation time of the study.

The results of the univariate and logistic regression analysis are shown in Tables II, III, respectively. In the univariate analyses, the cases and control groups differed significantly in the following areas of their medical histories: nephropathy $(\mathrm{p}<0.001)$, diabetes mellitus $(\mathrm{p}=$ $0.05)$, prior antibiotic use $(p=0.001)$, vancomycin use $(p$ $=0.02)$ and carbapenem use $(p<0.001)$. In the multivariate analysis, significant independent risk factors for VRE colonisation were nephropathy ( $p<0.001)$, prior antibiotic use $(p=0.03)$ and carbapenem use $(p<0.001)$.

The consumption of vancomycin, carbapenems and third and fourth generation cephalosporins in the ICU represented by the DDD per 1,000 patients-day varied during the study period (Fig. 1). On average, the DDD of cephalosporins, carbapenems and vancomycin were $589.33 / 1,000,243.53 / 1,000$ and $152.78 / 1,000$ patientsday, respectively. Despite the high density of cephalosporin use, only the use of vancomycin (Fig. 2) and carbapenems (Fig. 3) was related to the increased incidence of VRE-colonisation per 1,000 patients-day, according to the Pearson's correlation ( $r=0.61, p=0.03$ and $\mathrm{r}=0.53, \mathrm{p}=0.05$, respectively).

\section{DISCUSSION}

This study of ICU patients found a high prevalence of VRE vanC colonisation among patients admitted to the ICU (23.1\%). Prior antibiotic use, including car- bapenem and vancomycin use in the ICU, as well as diabetes mellitus and nephropathy, were associated with VRE colonisation in ICU patients by univariate analysis. Despite the high rates of colonisation by vanC VRE enterococci among hospitalised patients, no patients infected with bacteria of these phenotypes were detected in the ICU in this study. However, it is important to report that during the study period, two strains of E. gallinarum-causing bacteraemia were isolated in patients admitted to other hospital units, illustrating their role as potential opportunistic pathogens (Reid et al. 2001, Dargere et al. 2002, Choi et al. 2004).

Patients colonised by vanC VRE are a potential epidemiologic reservoir of resistance genes. In Brazil, the first case of colonisation by E. gallinarum carrying the van $\mathrm{A}$ gene was detected in 2002 in a hospital located in the state of Rio Grande do Sul (Camargo et al. 2004) and the first case of a clinical infection was detected in 2002 in the state of Rio de Janeiro (Merquior et al. 2008). During routine colonisation surveillance in the same hospital in RJ, seven vanA-carrying E. gallinarum isolates were detected (Neves et al. 2009).

Therapy with various antimicrobial agents may contribute to increasing colonisation by vanC VRE and others multiresistant microorganisms (Neves et al. 2009). Several studies have concluded that the consumption of antimicrobial agents is a strong indicator for multiresistant microorganism acquisition (Harthug et al. 2002). In our casuistic study, the use of antibiotics was present in approximately $70 \%$ of the 333 patients (223 patients) included in this study; most of these were broad spectrumcephalosporins (50.4\%), carbapenems (34.2\%) and vancomycin (26.1\%). This overuse of antibiotics, expressed as the DDD per 1,000 patients-day, reflects the high frequency of patients with infection in the ICU, although the majority of them were receiving empirical therapy with two drugs. Organisms isolated from patients in ICUs are more likely to be resistant to antibiotics than those isolated from general ward patients or outpatients (Archibald et al. 1997) because there is high antimicrobial selection pressure in these units (Vincent 2003).

TABLE I

Species distribution of enterococci species according to resistance

\begin{tabular}{lcccccccccc}
\hline & \multicolumn{1}{c}{ Antimicrobial n (\%) } \\
\cline { 2 - 11 } Species (number tested) & RIF & CLO & VAN & ERY & PEN & CIP & TET & AMP & HLGR & HLSR \\
\hline Enterococcus faecium (4) & $1(25)$ & $0(0)$ & $0(0)$ & $2(50)$ & $0(0)$ & $2(50)$ & $2(50)$ & $0(0)$ & $0(0)$ & $1(25)$ \\
Enterococcus faecalis (11) & $0(0)$ & $3(27.3)$ & $1(9.1)$ & $9(81.8)$ & $2(18.2)$ & $6(54.5)$ & $5(45.4)$ & $0(0)$ & $2(18.2)$ & $1(9.1)$ \\
Enterococcus gallinarum (26) & $0(0)$ & $1(3.8)$ & $0(0)$ & $6(23)$ & $0(0)$ & $3(11.5)$ & $8(30.8)$ & $0(0)$ & $0(0)$ & $0(0)$ \\
Enterococcus casseliflavus (51) & $1(2)$ & $6(11.8)$ & $0(0)$ & $25(49)$ & $0(0)$ & $5(9.8)$ & $15(29.4)$ & $0(0)$ & $1(2)$ & $10(19.6)$ \\
\hline Total (92) & $2(2.2)$ & $10(10.9)$ & $1(1.1)$ & $42(45.7)$ & $2(2.2)$ & $16(17.3)$ & $30(32.6)$ & $0(0)$ & $3(3.3)$ & $12(13)$ \\
\hline
\end{tabular}

AMP: ampicillin; CIP: ciprofloxacin; CLO: chloramphenicol; ERY: erythromycin; HLGR: high level gentamicin resistance; HLSR: high level streptomycin resistance; PEN: penicillin; RIF: rifampin; TET: tetracycline; VAN: vancomycin. 
TABLE II

Risk factors for vancomycin-resistant Enterococcus spp in hospitalized patients

\begin{tabular}{|c|c|c|c|}
\hline \multirow[b]{2}{*}{ Risk factors } & \multicolumn{2}{|c|}{ Frequency $(\%)$ or mean $\pm \mathrm{SD}$} & \multirow{2}{*}{$\begin{array}{c}\text { Univariate analysis } \\
\mathrm{p} \\
(\mathrm{OR} ; 95 \% \mathrm{CI})\end{array}$} \\
\hline & $\begin{array}{l}\text { Cases } \\
(\mathrm{n}=21)\end{array}$ & $\begin{array}{l}\text { Controls } \\
(n=143)\end{array}$ & \\
\hline Age (years) & $52.9( \pm 17.6)$ & $50.5( \pm 18.9)$ & 0.54 \\
\hline \multicolumn{4}{|l|}{ Gender } \\
\hline Female & 38.1 & 41.3 & $0.78(0.87 ; 0.34-2.24)$ \\
\hline Male & 61.9 & 58.7 & $0.78(1.14 ; 0.44-2.93)$ \\
\hline Duration of hospitalization (days) & $7.4( \pm 4.9)$ & $5.8( \pm 3.9)$ & 0.18 \\
\hline Prior ICU hospitalization & 71.4 & 59.4 & $0.29(1.70 ; 0.62-4.66)$ \\
\hline Surgery & 52.4 & 53.1 & $0.94(0.97 ; 0.39-2.43)$ \\
\hline \multicolumn{4}{|l|}{ Underlying diseases } \\
\hline Cancer & 14.3 & 9.8 & $0.46(1.54 ; 0.40-5.87)$ \\
\hline Diabetes mellitus & 28.6 & 12.6 & $0.05(2.74 ; 1.01-8.08)$ \\
\hline Cardiac disease & 42.9 & 50.3 & $0.52(0.74 ; 0.29-1.86)$ \\
\hline Autoimmune disease & 4.8 & 2.8 & $0.50(1.74 ; 0.18-16.34)$ \\
\hline Nephropathy & 23.8 & 4.2 & $<0.001(7.13 ; 1.95-26.06)$ \\
\hline \multicolumn{4}{|l|}{ Antibiotics treatment } \\
\hline Prior antibiotic use & 23.8 & 4.9 & $0.001(6.38 ; 1.81-22.48)$ \\
\hline Vancomycin & 19 & 4.2 & $0.02(5.37 ; 1.38-20.98)$ \\
\hline Third or fourth generation cephalosporin & 38.1 & 27.3 & $0.30(1.64 ; 0.63-4.26)$ \\
\hline Fluoroquinolones & 9.5 & 5.6 & $0.62(1.77 ; 0.35-8.99)$ \\
\hline Carbapenems & 28.6 & 2.8 & $<0.001(13.90 ; 3.52-54.87)$ \\
\hline Overall mortality & 14.3 & 9 & $0.44(1.63 ; 0.43-6.29)$ \\
\hline
\end{tabular}

CI: confidence interval; ICU: intensive care unit; OR: odds ratio; SD: standard deviation.

TABLE III

Logistic regression of risk factors for vancomycin-resistant Enterococcus spp in hospitalized patients

\begin{tabular}{lcc}
\hline Risk factors & $\mathrm{p}$ & OR $(95 \% \mathrm{CI})$ \\
\hline Underlying disease & & \\
$\quad$ Diabetes mellitus & 0.79 & $1.22(0.26-5.82)$ \\
$\quad$ Nephropathy & $<0.001$ & $13.65(3.24-57.55)$ \\
Antibiotics & & \\
$\quad$ Prior antibiotic use & 0.03 & $5.52(1.08-28.05)$ \\
$\quad$ Vancomycin & 0.54 & $1.70(0.30-9.67)$ \\
Carbapenems & $<0.001$ & $17.29(3.42-87.56)$ \\
\hline
\end{tabular}

CI: confidence interval; OR: odds ratio.

The consumption of third and fourth-generation cephalosporins, vancomycin and carbapenems in our ICU is much higher than that in many other countries (Moreira et al. 2009). The use of antibiotics, particularly vancomycin, has been identified as a risk factor for VRE colonisation or infection in some, but not all studies (Fridkin et al. 2001, Furtado et al. 2005, 2006, Sakka et al. 2008). Accordingly, the positive correlation in our study between the incidence of VRE and the consumption of vancomycin and carbapenems in the ICU suggest that the prevalence of antibiotic use is an important risk factor, particularly for the emergence and spread of VRE.

It has been reported that vancomycin use maintains the intestinal environment in a state that is favourable for VRE growth and promotes the possibility of an individual VRE carrier becoming a transmitter, additionally increasing the risk of a non-carrier becoming colonised (Furtado et al. 2006). Treatment with vancomycin was more common in VRE-colonised patients in the present study, but this association was not statistically significant in multivariate models. Unlike vancomycin use, carbapenem use in the ICU was an independent risk factor for VRE colonisation. Recent studies suggested that some carbapenems are excreted in high concentrations in the bile, resulting in the inhibition of anaerobes and overgrowth of enterococci (Stiefel et al. 2007).

Apart from the use of antimicrobial agents and inpatient care in particular units, other risk factors for VRE colonisation and infection that have been identified in other studies with samples of vanA VRE include the following: length of hospital stay, solid organ transplants, chronic dialysis, admission to the hospital at least once during the 12 months prior to ICU admission and hospitalisation for over three days prior to ICU admission (Ostrowsky et al. 1999, Warren et al. 2003, Furtado et al. 2005). We found that nephropathy was a risk factor for VRE colonisation. Patients with nephropathy are at 


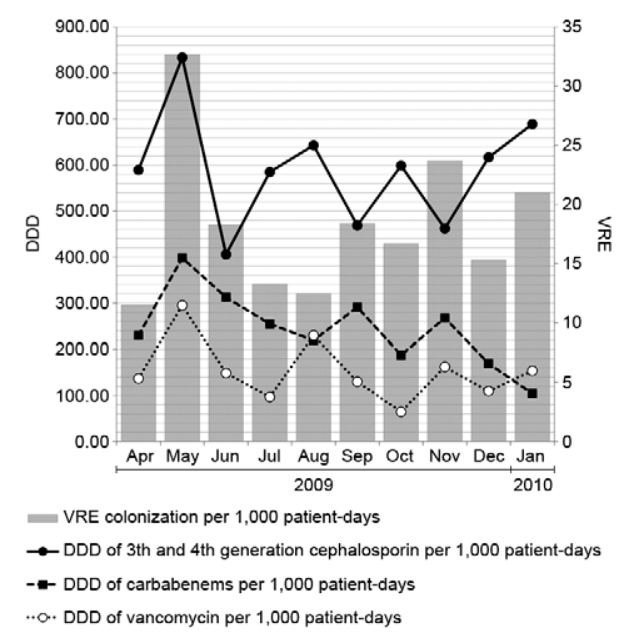

Fig. 1: relationship between the vancomycin-resistant enterococci (VRE) colonization per 1,000 patient-days (gray columns) and the density of third and fourth generation cephalosporins (filled circles), carbapenems (filled squares) and vancomycin (empty circles) use expressed as defined daily dose (DDD) per 1,000 patient-days in adult intensive care units from April 2009-January 2010.

an increased risk for acquiring VRE for several reasons, such as extensive contact with the hospitals, presence of multiple comorbid conditions, need for haemodialysis, proximity to other VRE-colonised patients, or even infected patients receiving prolonged administration of antibiotics, including vancomycin (Humphreys et al. 2004, Assadian et al. 2007).

The presence of VRE in infections is more common in ICUs in North America and in parts of South America than in European ones (Guzman-Blanco et al. 2000, Mutnick et al. 2003). VRE isolation has been reported in some Brazilian hospitals, mainly in the state of São Paulo (Cereda et al. 1997); in our hospital, VRE was described for the first time in 2003 in a post-surgical infection caused by an E. faecalis of vanA phenotype and was the first case to be described in MG (Ribas et al. 2007). However, high level aminoglycoside resistance has not been uncommon and frequencies of $18.5 \%$ in SP (Maschieto et al. 2004), 22\% in Brasília (Titze-deAlmeida et al. 2004) and 40.5\% in RJ (Mondino et al. 2003) have been observed. In the present study in Uberlândia, $16.3 \%$ of the isolates cultured from colonised individuals showed high levels of aminoglycoside resistance. An increase in the frequency of enterococci with high level aminoglycoside resistance has recently been observed due to spread of plasmids and transposons that encode enzymes that inactivate aminoglycosides (Sood et al. 2008, Protonotariou et al. 2010).

Several potential limitations of the present study should be mentioned. First, we had a relatively small number of patients in the case group, thus reducing the statistical power and the ability to study subsets of patients. Another limitation of this study was that information on the management of the patient's condition and severity of the underlying illness (e.g., the APACHE II

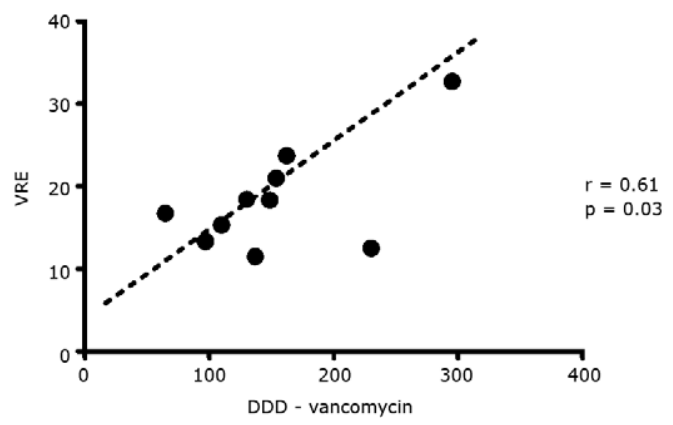

Fig. 2: Pearson's correlation coefficient between vancomycin-resistant enterococci (VRE) colonization per 1,000 patients-day and defined daily doses (DDD) of vancomycin per 1,000 patients-day.

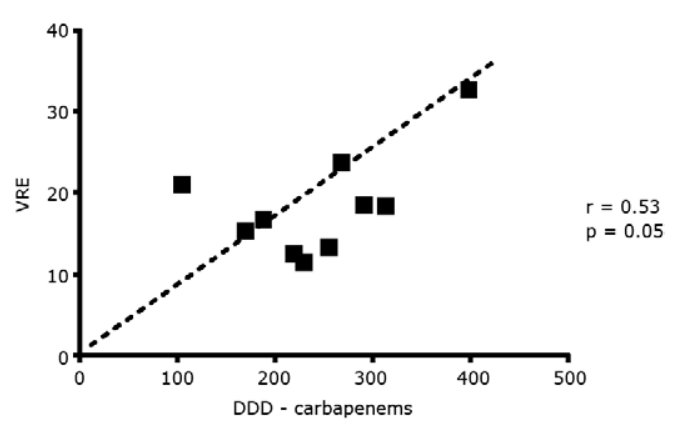

Fig. 3: Pearson's correlation coefficient between vancomycin-resistant enterococci (VRE) colonization per 1,000 patients-day and defined daily doses (DDD) of carbapenems per 1,000 patients-day.

score) was unavailable and the analysis of comorbid conditions was limited by the lack of data on the patient's underlying disease status.

In conclusion, our results showed a higher frequency of colonisation by VRE vanC, without an associated infection, associated with several risk factors, such as prior antibiotic use, carbapenem use and nephropathy as comorbidity. This study documents the emerging trend that the rates of VRE colonisation and administration of antibiotics (high DDD of antimicrobial) are very common and it may provide an accurate view of the problem in the ICUs of Brazilian university hospitals.

Despite several potential limitations, this study is the first to demonstrate the risk factors associated with vanC VRE colonisation in ICU hospitalised patients. Although van A and vanB enterococci have the greatest clinical importance, vanC VRE epidemiology should be better understood. Despite its indefinite clinical relevance, these species are opportunistic pathogens and vanC VRE-colonised patients are a potential epidemiologic reservoir of resistance genes.

\section{REFERENCES}

Archibald L, Phillips L, Monnet D, McGowan JE Jr, Tenover F, Gaynes R 1997. Antimicrobial resistance isolates from inpatients and outpatients in the United States: increasing importance of the intensive care unit. Clin Infect Dis 24: 211-215. 
Assadian O, Askarian M, Stadler M, Shaghaghian S 2007. Prevalence of vancomycin-resistant enterococci colonization and its risk factors in chronic haemodialysis patients in Shiraz, Iran. BMC Infect Dis 7: 52.

Camargo IL, Barth AL, Pilger K, Seligman BG, Machado AR, Darini AL 2004. Enterococcus gallinarum carrying the vanA gene cluster: first report in Brazil. Braz J Med Biol Res 37: 1669-1671.

Cereda R, Pignatari AC, Hashimoto A, Sader H 1997. In vitro antimicrobial activity against enterococci-isolated in a University Hospital in São Paulo-Brazil. J Infect Dis 1: 83-90.

Choi SH, Lee SO, Kim TH, Chung JW, Choo EJ, Kwak YG, Kim MN, Kim YS, Woo JH, Ryu J, Kim NJ 2004. Clinical features and outcomes of bacteraemia caused by Enterococcus casseliflavus and Enterococcus gallinarum: analysis of 56 cases. Clin Infect Dis 38: 53-61.

CLSI - Clinical Laboratory and Standards Institute 2009. Performance standards for antimicrobial susceptibility testing. Nineteenth Informational Supplement M100-S19, CLSI, Wayne, 177 pp.

Dargere S, Vergnaud M, Verdon R, Saloux E, Le Page O, Leclercq R, Bazin C 2002. Enterococcus gallinarum endocarditis occurring on native heart valves. J Clin Microbiol 40: 2308-2310.

de Perio MA, Yarnold PR, Warren J, Noskin GA 2006. Risk factor and outcomes associated with non-Enterococcus faecalis, nonEnterococcus faecium enterococcal bacteraemia. Infect Control Hosp Epidemiol 27: 28-33.

Fridkin SK, Edwards JR, Courval JM, Hill H, Tenover FC, Lawton R, Gaynes RP, McGowan JE Jr, Intensive Care Antimicrobial Resistance Epidemiology (ICARE) Project, the National Nosocomial Infections Surveillance (NNIS) System Hospitals 2001. The effect of vancomycin and third generation cephalosporins on prevalence of vancomycin-resistant enterococci in 126 USA adult intensive care units. Ann Intern Med 135: 175-183.

Furtado GH, Martins ST, Coutinho AP, Wey SB, Medeiros EA 2005. Prevalence and factors associated with rectal vancomycin-resistant enterococci colonization in two intensive care units in São Paulo, Brazil. Braz J Infect Dis 9: 64-69.

Furtado GH, Mendes RE, Pignatari AC, Wey SB, Medeiros EA 2006. Risk factors for vancomycin-resistant Enterococcus faecalis bacteremia in hospitalized patients: an analysis of two case-control studies. Am J Infect Control 34: 447-451.

Guzman-Blanco M, Casellas JM, Sader HS 2000. Bacterial resistance to antimicrobial agents in Latin America. The giant is awakening. Infect Dis Clin North Am 14: 67-81.

Harthug S, Jureen R, Mohn SC, Digranes A, Simonsen GS, Sundsfjord A, Langeland N, Norwegian Enterococcal Study Group 2002. The prevalence of faecal carriage of ampicillin-resistant and high-level gentamicin-resistant enterococci among inpatients at 10 major Norwegian hospitals. J Hosp Infect 50: 145-154.

Hendrix CW, Hammond JM, Swoboda SM, Merz WG, Harrington SM, Perl TM, Dick JD, Borschel DM, Halczenko PW, Pelz RK, Rocco LE, Conway JE, Brower RG, Lipsett PA 2001. Surveillance strategies and impact of vancomycin resistant enterococcal colonization and infection in critically ill patients. Ann Surg 233: 259-265.

Humphreys H, Dolan V, Sexton T, Conlon P, Rajan L, Creamer E, Walshe J, Donohoe J, Smyth EG 2004. Implications of colonization of vancomycin-resistant enterococci (VRE) in renal dialysis patients. Learning to live with it? J Hosp Infect 58: 28-33.

Maschieto A, Martinez R, Palazzo IC, Darini ALC 2004. Antimicrobial resistance of Enterococcus sp. isolated from the intestinal tract of patients from a university hospital in Brazil. Mem Inst Oswaldo Cruz 99: 763-767.
Mazuski JE 2008. Vancomycin-resistant enterococcus: risk factors, surveillance, infections and treatment. Surg Infect 9: 567-571.

Merquior VLC, Neves FPG, Ribeiro RL, Duarte RS, Marques EA, Teixeira LM 2008. Bacteraemia associated with a vancomycinresistant Enterococcus gallinarum strain harbouring both the van $\mathrm{A}$ and van $\mathrm{C} 1$ genes. J Med Microbiol 57: 244-245.

Metallidis S, Chatzidimitriou M, Tsona A, Bisiklis A, Lazaraki G, Koumentaki E, Gikas A, Alexiou-Daniel S, Nikolaidis P 2006. Vancomycin-resistant enterococci, colonizing the intestinal tract of patients in a university hospital in Greece. Braz J Infect Dis 10: 179-184.

Mondino SS, Castro AC, Mondino PJ, Carvalho M da G, Silva KM, Teixeira LM 2003. Phenotypic and genotypic characterization of clinical and intestinal enterococci isolated from inpatients and outpatients in two Brazilian hospitals. Microb Drug Resist 9: 167-174.

Moreira MR, Ribas RM, Rodrigues AAA, Gontijo Filho PP 2009. Consumo de antibióticos e etiologia de pneumonia associada à ventilação em pacientes internados na unidade de terapia intensiva do Hospital de Clínicas da Universidade Federal de Uberlândia. Rev Panam Infectol 11: 11-16.

Mutnick AH, Biedenbach DJ, Jones RN 2003. Geographic variations and trends in antimicrobial resistance among Enterococcus faecalis and Enterococcus faecium in the SENTRY Antimicrobial Surveillance Program (1997-2000). Diag Microbiol Infect Dis 46: 63-68.

Neves FP, Ribeiro RL, Duarte RS, Teixeira LM, Merquior VL 2009. Emergence of the vanA genotype among Enterococcus gallinarum isolates colonizing the intestinal tract of patients in a university hospital in Rio de Janeiro, Brazil. Int J Antimicrob Agents 33: 211-215.

Ostrowsky BE, Venkataraman L, D'Agata EM, Gold HS, De Girolami PC, Samore MH 1999. Vancomycin-resistant enterococci in intensive care units: high frequency of stool carriage during a non-outbreak period. Arch Intern Med 159: 1467-1472.

Protonotariou E, Dimitroulia E, Pournaras S, Pitiriga V, Sofianou D, Tsakris A 2010. Trends in antimicrobial resistance of clinical isolates of Enterococcus faecalis and Enterococcus faecium in Greece between 2002 and 2007. J Hosp Infect 3: 225-227.

Reid KC, Cockerill III FR, Patel R 2001. Clinical and epidemiological features of Enterococcus casseliflavus/flavescens and Enterococcus gallinarum bacteraemia: a report of 20 cases. Clin Infect Dis 32: 1540-1546.

Ribas RM, Darini AL, Moreira TA, Freitas C, Gontijo Filho PP 2007. Vancomycin-resistant vanA phenotype Enterococcus faecalis: first case in Minas Gerais state and epidemiological considerations. Braz J Infec Dis 11: 439-440.

Sakka V, Tsiodras S, Galani L, Antoniadou A, Souli M, Galani I, Pantelaki M, Siafakas N, Zerva L, Giamarellou H 2008. Risk-factors and predictors of mortality in patients colonized with vancomycin-resistant enterococci. Clin Microbiol Infect 14: 14-21.

Sood S, Malhotra M, Das BK, Kapil A 2008. Enterococcal infections \& antimicrobial resistance. Indian J Med Res 128: 111-121.

Stiefel U, Pultz NJ, Donskey CJ 2007. Effect of carbapenem administration on establishment of intestinal colonization by vancomycin-resistant enterococci and Klebsiella pneumoniae in mice. Antimicrob Agents Chemother 51: 372-375.

Teixeira LM, Siqueira-Carvalho MG, Facklam RR 2007. Enterococcus. In PR Murray, EJ Baron, JH Jorgensen, ML Landry, MA Pfaller (eds.), Manual of clinical microbiology, ASM Press, Washington DC, p. 430-442.

Titze-de-Almeida R, Rollo Filho M, Nogueira CA, Rodrigues IP, Eudes Filho J, Nascimento RS, Ferreira RF 2nd, Moraes LM, 
Boelens H, Van Belkum A, Felipe MS 2004. Molecular epidemiology and antimicrobial susceptibility of enterococci recovered from Brazilian intensive care units. Braz J Infect Dis 8: 197-205.

Toye B, Shymanski J, Bobrowska M, Woods W, Ramotar K 1997. Clinical and epidemiologic significance of enterococci intrinsically resistant to vancomycin (possessing the van $\mathrm{C}$ genotype) J Clin Microbiol 35: 3166-3170.

Vincent JL 2003. Nosocomial infections in adult intensive-care units. Lancet 361: 2068-2077.

Warren DK, Kollef MH, Seiler SM, Fridkin SK, Fraser VJ 2003. The epidemiology of vancomycin-resistant Enterococcus coloniza- tion in a medical intensive care unit. Infect Control Hosp Epidemiol 24: 257-263.

Werner G, Coque TM, Hammerum AM, Hope R, Hryniewicz W, Johnson A, Klare I, Kristinsson KG, Leclercq R, Lester CH, Lillie M, Novais C, Olsson-Liljequist B, Peixe LV, Sadowy E, Simonsen GS, Top J, Vuopio-Varkila J, Willems RJ, Witte W, Woodford N 2008. Emergence and spread of vancomycin resistance among enterococci in Europe. Euro Surveill 13: 1-11.

Woodford N, Morrison D, Johnson AP, George RC 1993. Antimicrobial resistance amongst enterococci isolated in the United Kingdom: a reference laboratory perspective. J Antimicrob Chemother 32: $344-346$ 